OPEN ACCESS

Edited by:

Changiz Mohiyeddini,

Oakland University William Beaumont

School of Medicine, United States

Reviewed by:

Guillermo Felipe López Sánchez,

Anglia Ruskin University,

United Kingdom

Andrea Sabrina Hartmann,

University of Osnabrück, Germany

*Correspondence:

Nada M. Albawardi

nbwardi@yahoo.com

Hazzaa M. Al-Hazzaa

halhazzaa@hotmail.com

Specialty section:

This article was submitted to

Health Psychology,

a section of the journal

Frontiers in Psychology

Received: 29 September 2020

Accepted: 15 January 2021

Published: 11 February 2021

Citation:

Albawardi NM, AlTamimi AA,

AlMarzooqi MA, Alrasheed $L$ and Al-Hazzaa HM (2021) Associations of Body Dissatisfaction With Lifestyle

Behaviors and Socio-Demographic

Factors Among Saudi Females

Attending Fitness Centers.

Front. Psychol. 12:611472.

doi: 10.3389/fpsyg.2021.611472

\section{Associations of Body Dissatisfaction With Lifestyle Behaviors and Socio-Demographic Factors Among Saudi Females Attending Fitness Centers}

\section{Nada M. Albawardi' ${ }^{*}$, Abeer A. AlTamimi², Mezna A. AlMarzooqi ${ }^{3}$, Lama Alrasheed ${ }^{1}$ and Hazzaa M. Al-Hazzaa ${ }^{\text {* }}$}

'Lifestyle and Health Research Center, Health Sciences Research Center, Princess Nourah Bint Abdulrahman University, Riyadh, Saudi Arabia, ${ }^{2}$ Health Sciences Research Center, Princess Nourah Bint Abdulrahman University, Riyadh, Saudi Arabia, ${ }^{3}$ Department of Community Health Sciences, College of Applied Medical Sciences, King Saud University, Riyadh, Saudi Arabia

Objective: To examine body image perception and the associations of body dissatisfaction (BD) with socio-demographic and lifestyle factors among Saudi women attending fitness centers in Riyadh.

Methods: Saudi females aged 16 years and older were recruited from 12 randomly selected fitness centers in Riyadh, using stratified clustered sampling technique $(n=460)$. Height and weight were measured to calculate actual body mass index (BMI). A previously validated instrument was used to collect socio-demographic and lifestyle variables including physical activity (PA), sedentary behaviors, sleep and dietary habits. Stunkard Figure Rating Scale silhouettes were used to assess perceived and desired body shape.

Results: The participants had a mean (SD) age of 29.2 (8.2). The majority were not married (57\%), with no children (66\%) and had college degrees (78\%). While 63\% were overweight or obese, nearly $40 \%$ of women underestimated their perceived body shape. The majority of respondents (87\%) were dissatisfied with their body shape including $68 \%$ of normal weight women. Females who had BD were significantly older, had higher BMl, reported more weight loss attempt and had expended less time in vigorous $(p=0.033$ ) and total $(p=0.042)$ PA than those who were satisfied with their body shape. However, when adjusting for socio-demographic variables, logistic regression analysis revealed significant associations of BD with higher BMl, shorter membership duration of fitness club, and reduced dairy products and energy drinks consumption.

Conclusion: Except for BMl and decreased dairy products and energy drinks consumption, many lifestyle behaviors did not associate with BD among Saudi females attending fitness centers. The findings can inform healthcare providers when 
intervention strategy is implicated for females with BD. Future studies should compare the associations of BD with lifestyle behaviors between males and females attending fitness centers and seeking weight loss.

Keywords: body image, perception, body mass index, females, obesity, lifestyle behaviors, Saudi Arabia, body image dissatisfaction

\section{INTRODUCTION}

The escalating public health concerns relating to obesity and its associated health consequences have led to a plethora of interventions designed to address the growing risk factors of physical inactivity, malnutrition, and eating disorders. These interventions include strategies to affect positive behavioral changes using both conscious, decision-based models as well as models that address non-conscious processes (Rejeski and Fanning, 2019). The effectiveness of such programs is often moderated by a number of variables including psychosocial, biological and environmental factors (Rejeski and Fanning, 2019). Among the psychosocial factors; body image (BI) perception and body dissatisfaction (BD) have been shown to be associated with obesity (Anderson et al., 2002; Duong and Roberts, 2014; Jun and Choi, 2014), physical activity (PA) (Hausenblas and Fallon, 2006), dietary habits (Fan and Jin, 2015), eating disorders (Lawler and Nixon, 2011), and depression (Paans et al., 2018). BI is a multidimensional construct encompassing perceptual, attitudinal, and behavioral dimensions. Body image perception (BIP) is a component of the perceptual and behavioral dimension and relates to how one sees and describes their body in relation to body size, shape, and weight (Sabiston et al., 2019). BD is a component of the attitudinal dimension and refers to an undesirable subjective assessment of one's body often described as when desired BI does not match with the perceived BI.

Inaccurate perceptions of body size (BIP) are common particularly among children and adolescents (Neves et al., 2017). In a study of nearly 30,000 children and adolescents in Taiwan (Hsu et al., 2016), the prevalence of body weight misperception was $43.2 \%$ (26.4\% overestimation and $16.8 \%$ underestimation). Similarly, in a sample of 4979 adolescents in the in the United Kingdom, 39\% of those overweight/obese ( $47 \%$ of boys, $32 \%$ of girls) underestimated themselves as "about the right weight" or even "too light" (Jackson et al., 2015). Likewise, over $45 \%$ of overweight/obese subjects from a sample of 660 adolescents from the United Arab Emirates believed they were normal weight (Musaiger et al., 2012). While fewer studies report on the accuracy of adults body weight estimations, misperceptions clearly exist. Among 1,295 Dutch older adults, only $4.4 \%$ of obese men and $12.3 \%$ of obese women accurately perceived their body weight status (Monteagudo et al., 2015). Similarly, in a large sample of overweight/obese young adults in the United States, 20\% of females and $48 \%$ of males under-estimated their weight (Sonneville et al., 2016). Saudi college aged women with overweight/obesity also underestimated their actual body mass index (BMI) by $8.5 \%(-2.5 \pm 2.5 \mathrm{~kg} / \mathrm{m} 2)$, while those without overweight/obesity slightly overestimated their BMI by $1.9 \%$ (Albeeybe et al., 2018).

Irrespective of actual weight status, both over and underestimation have often been found to negatively affect weight management and PA in adolescents (Jankauskiene and Baceviciene, 2019) and adults (Voelker et al., 2015). In a review of 74 studies on weight perception and associated weight management, Haynes et al. (2018) concluded that perceived overweight was not reliably associated with increased PA or healthy eating and was associated with greater disordered eating in some groups. Overestimation, while increasing the number of attempts at weight loss, has consistently been associated with increased likelihood of engaging in health compromising behaviors (Fan and Jin, 2015; Robinson et al., 2015). In addition, both over- and under-estimation of body weight have been associated with unhealthy weight control behaviors and depressive mood (Jankauskiene and Baceviciene, 2019). In a study of over 4000 Canadian adolescents, those perceiving themselves as overweight or obese had lower odds (OR: 0.59; 95\% CI: $0.42-0.81$ ) of adherence to PA recommendations compared with those who felt they were about the right weight (Sampasa-Kanyinga et al., 2017). Several longitudinal studies have also shown that perceiving oneself as overweight, regardless of actual size, increased the risk of future weight gain (Robinson et al., 2015; Haynes et al., 2018; Feng and Wilson, 2019). On the other hand, accurate estimation of excess weight was observed to have a positive effect on weight management in a 13 -year follow-up of obese women who perceived themselves as too large and showed less weight gain over time than those who underestimated their weight (Lynch et al., 2009). In addition decreased awareness of actual weight status may lead some to be less aware of their risk for negative health consequences (Miller et al., 2008). Thus it appears that body weight estimation may play both a negative and positive role in adopting healthy lifestyle behaviors (Jankauskiene and Baceviciene, 2019).

The health consequences that may result from the unique interaction of age, body mass, and $\mathrm{BD}$ are particularly relevant as correlates, precursors, and consequences of PA (Sabiston et al., 2019). A positive BI, rather than BMI, is often a stronger predictor of PA engagement (Kantanista et al., 2015; SampasaKanyinga et al., 2017). While BD is associated with lower PA, and dysfunctional exercise (Sampasa-Kanyinga et al., 2017; Jankauskiene and Baceviciene, 2019), participating in exercise has been shown to have a positive impact on BI (FernándezBustos et al., 2019). A meta-analysis on the effect of exercise on BI found exercisers had a more positive BI than nonexercisers (Hausenblas and Fallon, 2006). Exercise interventions also resulted in improved BI scores post-intervention compared to controls (Hausenblas and Fallon, 2006). Positive effects of 
engaging in PA on BI, with and without changes in body composition, are consistently demonstrated in the literature (Voelker et al., 2015).

Over the past decade, there has been accumulating evidence showing that body weight concerns and dissatisfaction are fairly prevalent among young Arab women. A recently published research involving young Arab females from five Arab countries including Bahrain, Egypt, Jordan, Oman, and Syria revealed that one third of the females were dissatisfied with their body weight status (Musaiger, 2014). Similarly Saudi females reported a notably higher proportion of BID (33.5\%) than males (21.4\%) (Al-Otaibi et al., 2013). Such findings may call attention to the sociocultural influences and media pressure that emphasized body thinness among Saudi young females (Albeeybe et al., 2018).

Few studies have investigated the associations between BI perception, $\mathrm{BD}$ and lifestyle variables. In particular, none of the previous studies had examined the associations of BD and lifestyle behaviors among females engaging in physical activities while mostly seeking body weight loss. Local studies are scarce and either did not focus primarily on the association of BI and lifestyle, used small sample sizes or did not use validated PA or BI measures (Ben-Ammar and Al-Holy, 2013; Alhussaini et al., 2018). In addition, due to the recent social and political changes in Saudi Arabia, there has been a surge in female fitness centers and increased engagement of females in PA, especially those with excess body weight (AlTamimi et al., 2020). Consequently, studying females participating in fitness centers may help in understanding the associations between body satisfaction or dissatisfaction and lifestyle behaviors and socio-demographic factors. Such approach may enhance our understanding of the different factors that promote body satisfaction in females and is an important tool to facilitating the adoption of healthy lifestyle behaviors. Therefore, the aim of the present study is to investigate the status of BI perception and to explore the associations of BD with demographics, PA, screen time, dietary habits and sleep among adult females attending fitness centers in Riyadh, the capital of Saudi Arabia.

\section{MATERIALS AND METHODS}

\section{Participants}

Twelve fitness centers in Riyadh, Saudi Arabia were randomly selected, using stratified (geographical locations) clustered sampling technique. Saudi females aged 16 years and older without any physical impairment were recruited from these centers using a predetermined sample size of 384 participants. The sample proportion was within 0.05 of the population proportion with a 95\% confidence level. The population proportion was assumed to be at 0.50 , as this proportion yields the maximal possible sample size required. An additional 20\% for clustered design effect and missing data was added resulting in a target sample size of 460 females. All female participants in the sampled fitness center were approached during the data collection period and asked to take part in the study. Visits to the fitness centers included random days during the week and on weekends. Ethical approval was attained from the ethical committee at the Health Sciences Research Center, Princess Nourah University (IRB Log Number: 18-0222). Written consent form was obtained from each of the participating females.

\section{Anthropometric Measurements}

Body weight was measured to the nearest $100 \mathrm{~g}$, with minimal clothing and without shoes, using a calibrated portable medical scale (Seca scale model 770, Seca, Hamburg, Germany). Height was measured to the nearest $\mathrm{cm}$ with the subject in the full standing position without shoes using calibrated portable measuring rod. BMI was calculated as body weight in $\mathrm{kg}$ divided by squared height in meters and then classified into "underweight," "normal," overweight," and "obese" according to World Health Organization (WHO) criteria (World Health Organization, 2000).

\section{Perceived and Desired Body Image Assessment}

Stunkard Figure Rating Scale (FRS) nine silhouette figures were used to assess perceived and desired BI (Stunkard et al., 1983). The perceived BI scores were classified into BMI categories according to Bulik et al. (2001), who established the equivalence between each silhouette and BMI in a study of 16,728 American women and 11, 366 men. Silhouettes were categorized as underweight (silhouette 1), normal weight (silhouette 2-4), overweight (silhouette 5 and 6), and obese (silhouette 7-9). The discrepancy between the actual BMI category and the BMI category corresponding to the perceived BI score represents the accuracy of the respondents BI perception. Accuracy of BIP was stratified into "overestimation" (perceived > actual BMI category), "accurate" (perceived = actual BMI category), and "underestimation" (perceived < actual BMI category).

Second, the discrepancy between perceived and desired BI scores was calculated to represent the individual's satisfaction with their BI. Respondents were categorized as "dissatisfied" if they desired a larger or smaller figure than their perceived image and "satisfied" if the perceived score was the same as the desired score.

\section{Physical Activity, Screen Time, and Sleep Duration Assessment}

The Arab Teen Lifestyle Study (ATLS) questionnaire was used to collect data related to PA, screen time, dietary habits, and sleep duration (Al-Hazzaa and Musaiger, 2011; Al-Hazzaa et al., 2011a). The questionnaire collects information on the frequency, duration, and intensity of several domains of activity including transport, household, fitness and sporting and leisure-time activities of light-, moderate-, and vigorous-intensity during a typical week. PA time per week was converted to activity energy expenditure values in METs-min/week, based on compendium of PA for adults (Ainsworth et al., 2011). The minimum activity energy expenditure was computed as 600 METs-min/week [equivalent to $150 \mathrm{~min}$ of moderate (four METs) per week]. In addition, participants reported on the typical time in hours spent per day on screen activities, including television (TV) 
viewing, video games, and computer and internet use during both weekdays and weekends. Low and high screen time cutoff values were based on below or above $2 \mathrm{~h}$ of screen viewing per day. The typical sleep durations at night in hours spent on weekdays and weekends were also reported by the participants. Insufficient sleep was defined as sleeping less than $7 \mathrm{~h}$ per night, according to the definition of the National Sleep Foundation for adults (Hirshkowitz et al., 2015). Previous studies have shown this questionnaire to be valid and reliable for assessing PA and other lifestyle habits in adolescents and young adults (Al-Hazzaa and Al-Ahmidi, 2003; Al-Hazzaa et al., 2011b).

\section{Data and Statistical Analysis}

Data was entered into an SPSS data file, checked, cleaned, and analyzed using SPSS program, version 22 (IBM, Chicago, IL, United States). Descriptive statistics were calculated and presented as means and standard deviations (SD) or frequencies and proportions. Bivariate analysis comparing the participants who were "satisfied" with their shape with those who were "dissatisfied and desired a smaller shape" was conducted using Chi-Square or T-tests as appropriate. Partial correlation coefficients (Pearson), while controlling for age, was used to test the relationships between perceived $\mathrm{BI}$, desired $\mathrm{BI}$ and $\mathrm{BD}$ with selected lifestyle behaviors. Multivariable logistic regression were used to examine the associations between BI satisfaction and dissatisfaction with levels of accuracy of BI perception (underestimated, accurately estimated, and overestimated BI), socio-demographic characteristics and engagement in lifestyle behaviors, specifically related to dietary intake, PA, and sleep. In all of the tests performed, we used alpha level of $<0.05$ as the level of significance.

\section{RESULTS}

After approaching 517 female participants during both weekdays and weekends, 460 agreed to take part in the study and this number constituted the final sample size, constituting a response rate of $89 \%$. Mean age (SD) of the sampled females was 29.2 (8.2) years ranging from 16 to 63 years. Body weight and height averaged $69.7(14.7) \mathrm{kg}$ and $158.7(5.5) \mathrm{cm}$, respectively, while the mean (SD) of BMI was $27.6(5.4) \mathrm{kg} / \mathrm{m}^{2}$ with $63 \%$ overweight or obesity prevalence. The majority of respondents were not married (57\%), did not have children (66\%) and had college degrees (78\%). Only $11 \%$ of women reported being "satisfied" with their body shape. The majority of women, 79\%, was "dissatisfied" and desired a smaller shape, while only $5 \%$ desired a larger shape. The mean perceived and desired BI scores, based on FRS were 4.5 (1.6) and $2.8(0.91)$, respectively. This means that the differences between perceived and desired BI scores was 1.7 (1.4) or $37.8 \%$. BMI corresponding to perceived and desired BI scores, based on BMI values corresponding to desired BI scores reported by Bulik et al. (2001) were 25.5 (4.9) and 20.8 (1.8), respectively. This is a difference of $18.4 \%$, while the difference between the actual BMI and BMI corresponding to perceived BI score was 7.9\%.

The socio-demographic characteristics of women who were "satisfied" and those "dissatisfied and desiring a smaller shape" are reported in Table 1. The mean BMI of those who were dissatisfied (28.7 \pm 5.1$)$ was significantly higher than those who were satisfied with their body shape $(23.3 \pm 3.2, p<0.001)$. The proportion of women with BD increased significantly with BMI categories (normal-61\%, overweight-95\%, obese- $98 \%$, $p<0.001)$. The participants' perception of their body shape was accurate for $52 \%$ of the respondents, while $40 \%$ underestimated their body shape, choosing a perceived body shape lower than that corresponding to their actual BMI. Dissatisfaction was significantly associated with weight loss attempts, with the proportion of women reporting BD increasing with increased weight loss attempts $(p<0.001)$. Duration of club membership was also significantly associated with $\mathrm{BD}(p=0.028)$.

Table 2 displays the association of lifestyle variables with subjects who were "satisfied" and those who were "dissatisfied and desired a smaller shape." The majority of the respondents screen time was over $2 \mathrm{~h}$ per day (89\%), however over 92\% participated in PA for 150 min per week or more. The "satisfied" group had significantly higher energy expenditure in METs$\mathrm{min} /$ week of vigorous and total activity $(p=0.033,0.042$, respectively). The average sleep time reported was $6.71 \pm 1.57 \mathrm{~h}$ per day with $46 \%$ of women sleeping $7 \mathrm{~h}$ or more per night. The mean frequency of eating breakfast, fruits, vegetables and dairy products was less than five times per week. Unhealthy foods were consumed an average of less than two times per week except for chocolates/candy (mean $2.7 \pm 2.3$ ).

Table 3 presents the partial correlation coefficients between perceived $\mathrm{BI}$, desired $\mathrm{BI}$, and $\mathrm{BD}$ with selected lifestyle behaviors, while controlling for age. Few correlations are significant. Perceived BI significantly correlated with screen time $(r=0.108$, $p=0.032)$, chocolates/candy intake $(r=-0.104, p=0.040)$, and energy drinks intake $(r=-0.102 ; p=-0.102, p=0.043)$. Desired BI significantly correlated with vigorous PA $(r=-0.138$, $p=0.020)$. Finally, BD correlated with chocolate/candy intake $(r=-0.124, p=0.015)$ and energy drinks intake $(r=-0.143$, $p=0.004)$.

Multivariable logistic regression analysis revealed four variables were predictors of BD (Table 4). Increases in BMI were predictive of increased likelihood of BD (OR: 1.73, CI: 1.38-2.16) while a lower odds of $\mathrm{BD}$ was found with consumption of dairy products (OR: 0.79, CI: 0.643-0.980) and energy drinks (OR: 0.26, CI: 0.099-0.679). In addition, club membership of 1-2 years reduced the likelihood of $\mathrm{BD}$ (OR: 0.27, CI: 0.078-0.948) as compared to less than 1 year of membership. Other dietary variables, PA, screen time, average sleep duration, and accuracy of BIP were not predictive of BD.

\section{DISCUSSION}

The present study evaluated BI through both a perceptual dimension, BIP, and an attitudinal dimension, BD, in a sample of Saudi women attending fitness centers in the capital of Saudi Arabia. This sample had a high proportion of overweight/obese women with high discrepancy between perceived and desired BI. The majority of the participants dissatisfied with their body and desiring a smaller shape. Those 
TABLE 1 | Association of body image dissatisfaction with socio-demographic variables and accuracy of perception of body shape among Saudi women attending fitness clubs in Riyadh, Saudi Arabia.

Variable All $\frac{\text { Body Image }}{\text { Satisfied Dissatisfied }^{1}}$

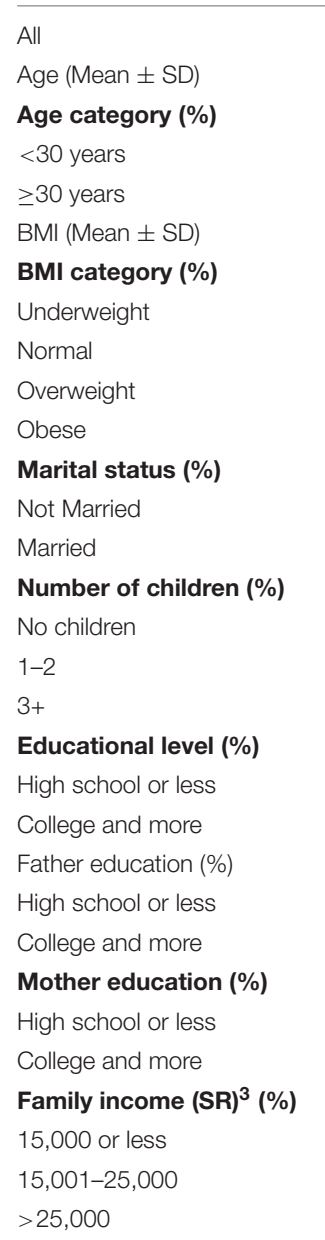

Club membership (\%)

Less than a year

$1-2$ years

3-4 years

5 years or more

Weight loss attempts (\%)

No attempt

1-2 attempts

3 attempts or more

Body image perception $(\%)^{4}$

Overestimate $\quad 28(6.8 \%) \quad 1(3.6 \%) \quad 27(96.4 \%)$

Accurate $\quad 216(52.2 \%) 42(19.4 \%) \quad 174(80.6 \%)$

Underestimate $\quad 167(40.3 \%) \quad 8(4.8 \%) \quad 159(95.2 \%)$

${ }^{1}$ Desired shape < perceived shape.

${ }^{2}$ Chi Squares tests for the differences in proportions, T-test for comparison of means (SD).

${ }^{3}$ US \$ $=3.75$ SR

${ }^{4}$ Body image perception: Overestimated = actual BMI category $<$ BMI category of perceived body shape. Accurate = actual BMI category $<$ BMI category of perceived body shape. Underestimated = actual BMI category $>$ BMI category of perceived body shape. satisfied and dissatisfied with their body shape did not differ significantly in their socio-demographic variables, sleep duration or screen time. Vigorous PA was significantly greater among those who were satisfied. However, when adjusting for sociodemographic variables, multivariable logistic regression analysis revealed significant associations of body shape dissatisfaction with higher BMI, shorter membership duration of fitness club, and reduced dairy products and energy drinks consumption.

Our findings showed that the majority $(63 \%)$ of our participants are considered in the overweight or obese category. Such rate is similar to the prevalence of overweight plus obesity (61.5\%) for Saudi women 15 years and older reported in a recent national survey (Memish et al., 2014). It is therefore not surprising that a relatively high proportion (47\%) of the participants misperceived their body shape. This is however similar to most studies that have found underestimation of body weight to be common among adults, particularly those who are overweight/obese (Dorsey et al., 2009; Herman et al., 2013; Clark et al., 2017). In Saudi Arabia, a study of over 900 female university students found $63 \%$ of women with overweight/obesity underestimated their actual BMI by $8.5 \%\left(-2.5 \pm 2.5 \mathrm{~kg} / \mathrm{m}^{2}\right)$ (Albeeybe et al., 2018). As $92 \%$ of our participants reported over $150 \mathrm{~min} /$ week of PA, another possible explanation is that studies have found overweight/obese individuals may be more likely to underestimate their weight if they are physically active. Among over 4,000 adults screened for stroke risk, obese subjects who exercised sufficiently showed greater odds of inaccurately perceiving themselves as normal weight as compared to those who were sedentary ( $\mathrm{OR}=0.45, \mathrm{CI}=0.35-0.57)$ (Miller et al., 2008). Likewise, a large study of adult Danes found overweight subjects with higher levels of leisure-time PA were twice as likely to misperceive their weight in comparison with sedentary individuals (Matthiessen et al., 2014). Inaccurate perceptions of body size are common, particularly among children and adolescents (Hsu et al., 2016; Neves et al., 2017; López and Rodríguez Cabeo, 2020) and adults (Monteagudo et al., 2015; Sonneville et al., 2016). It was also shown that both over and underestimation to negatively affect weight management and PA in adolescents (Jankauskiene and Baceviciene, 2019) and adults (Voelker et al., 2015). However, in a review of large number of studies on weight perception and associated weight management, it was concluded that perceived overweight was not reliably associated with increased PA or healthy eating and was rather associated with greater disordered eating in some groups (Haynes et al., 2018). On the other hand, accurate estimation of excess weight was observed to have a positive effect on weight management in a 13-year follow-up among female obese adolescents (Lynch et al., 2009).

This study found a high proportion of women with dissatisfaction of body shape (87\%) even among normal weight respondents. Previous studies showed that $\mathrm{BD}$ is common, particularly among females, both in adolescence and adulthood (Yager and O'Dea, 2008; Fan and Jin, 2015; Voelker et al., 2015; Allen and Walter, 2016; Neves et al., 2017). Similar results were found among Spanish adults $(n=1,081)$, with $67.1 \%$ of males and $81.0 \%$ females reporting BD (Bibiloni et al., 2017). Likewise, among over 15,000 respondents in the European 
Union, $54 \%$ of males and $69 \%$ of females were found to have BD (McElhone et al., 1999).

Among 336 mainly unmarried young adult female university students in the capital, Riyadh, $17 \%$ of normal BMI subjects, half of overweight (51\%) and the majority of obese subjects (70\%) were dissatisfied with their body shape (Alhussaini et al., 2018). Other studies in the Arab world, however, have shown lower rates of BD. Only a third of college females in five Arab countries (Bahrain, Egypt, Jordan, Oman, and Syria) were dissatisfied with their body shape (Musaiger, 2014), however the body weight of subjects was not reported. Likewise, in a study conducted on 368 university students from the East of Saudi Arabia, only a third of females $(33.5 \%)$ and $21.4 \%$ of males were found to have $\mathrm{BD}$, however this may be due to the majority of respondents (65\%) having normal BMI (Al-Otaibi et al., 2013).

This study found BMI, period of membership in the gym, and frequency of consumption of dairy products and energy drinks significant predictors for $\mathrm{BD}$. Increased $\mathrm{BMI}$ is a consistent predictor of BD among adolescents (Gouveia et al., 2014; Fernández-Bustos et al., 2019; Jankauskiene and Baceviciene, 2019), adults and even children (Neves et al., 2017). Findings from a 10-year longitudinal study in the United States reported a trend among youth of increasing BD as their BMI increased from middle school to young adulthood (Bucchianeri et al., 2013; Feng and Wilson, 2019). Likewise, a longitudinal study of Australian adults found BMI tended to be significantly higher among subjects with BD (Feng and Wilson, 2019). Similarly, data from Netherlands Study of Depression and Anxiety found higher BMI was significantly associated with greater BD independent of depression (Paans et al., 2018).

Body dissatisfaction may be seen as a natural consequence of increased BMI and a precursor for positive health behaviors. It is often proposed that accurate perception of one's body is vital for motivating weight loss intention and weight loss attempts, particularly among overweight and obese individuals (Baranowski et al., 2003; Rancourt et al., 2017). However, there is strong evidence from longitudinal studies that perceived overweight and BI dissatisfaction are associated with greater weight gain and increased likelihood of overweight/obesity onset over time (Robinson et al., 2015; Haynes et al., 2018), regardless of weight status at baseline and whether the subject's perception is accurate or inaccurate. Longitudinal studies suggest that BD among overweight adolescents is predictive of negative health consequences including physical inactivity, lower fruit/vegetables

TABLE 2 | Association of body image dissatisfaction with lifestyle variables among Saudi women attending fitness clubs in Riyadh, Saudi Arabia.

\begin{tabular}{|c|c|c|c|c|}
\hline \multirow[t]{2}{*}{ Variable } & \multirow[t]{2}{*}{ All } & \multicolumn{2}{|c|}{ Body Image } & \multirow[t]{2}{*}{$p$-Value ${ }^{2}$} \\
\hline & & Satisfied & Dissatisfied $^{1}$ & \\
\hline Sleep duration (\%) & & & & 0.499 \\
\hline Insufficient sleeper (<7 h) & $224(54.4 \%)$ & $26(11.6 \%)$ & $198(88.4 \%)$ & \\
\hline Sufficient sleeper ( $\geq 7$ h) & $188(45.6 \%)$ & $26(13.8 \%)$ & $162(86.2 \%)$ & \\
\hline Average sleep duration (hours/day)-mean \pm SD & $6.70 \pm 1.57$ & $7.09 \pm 1.45$ & $6.65 \pm 1.58$ & 0.056 \\
\hline Screen time $(\%)$ & & & & 0.790 \\
\hline Low Screen Time (<2 h/day) & 44 (10.7\%) & $5(11.4 \%)$ & $39(88.6 \%)$ & \\
\hline High Screen Time ( $\geq 2$ h/day) & $368(89.3 \%)$ & $47(12.8 \%)$ & $321(87.2 \%)$ & \\
\hline Average screen time (hours/day)-mean \pm SD & $4.46 \pm 2.21$ & $4.13 \pm 1.87$ & $4.51 \pm 2.26$ & 0.247 \\
\hline \multicolumn{5}{|l|}{ Food Frequency Intake - mean \pm SD } \\
\hline Breakfast intake (day/week) & $4.6 \pm 2.7$ & $4.9 \pm 2.7$ & $4.6 \pm 2.7$ & 0.388 \\
\hline Vegetables intake (day/week) & $4.7 \pm 2.1$ & $5.0 \pm 1.9$ & $4.7 \pm 2.2$ & 0.412 \\
\hline Fruit intake (day/week) & $3.7 \pm 2.3$ & $4.0 \pm 2.4$ & $3.7 \pm 2.3$ & 0.350 \\
\hline Milk and dairy products intake (day/week) & $4.7 \pm 2.3$ & $4.9 \pm 2.3$ & $4.7 \pm 2.3$ & 0.416 \\
\hline Sugar-sweetened drinks intake (day/week) & $1.5 \pm 1.8$ & $1.2 \pm 1.4$ & $1.6 \pm 1.9$ & 0.139 \\
\hline Fast foods intake (day/week) & $1.6 \pm 1.4$ & $1.5 \pm 1.2$ & $1.6 \pm 1.4$ & 0.675 \\
\hline French fries/potato chips intake (day/week) & $1.4 \pm 1.4$ & $1.0 \pm 1.1$ & $1.4 \pm 1.4$ & 0.087 \\
\hline Cake/donuts intake (day/week) & $1.9 \pm 1.8$ & $1.5 \pm 1.5$ & $2.0 \pm 1.9$ & 0.123 \\
\hline Chocolates/candy intake (day/week) & $2.7 \pm 2.2$ & $2.5 \pm 2.2$ & $2.7 \pm 2.2$ & 0.465 \\
\hline Energy drink intake (day/week) & $0.1 \pm 0.5$ & $0.2 \pm 0.8$ & $0.1 \pm 0.5$ & 0.056 \\
\hline Classification of PA Category (\%) & & & & 0.417 \\
\hline High activity time ( $\geq 150$ min/week) & $425(92.4 \%)$ & $48(12.4 \%)$ & $340(87.6 \%)$ & \\
\hline Low activity time (<150 min/week) & $35(7.6 \%)$ & $4(15.4 \%)$ & $22(84.6 \%)$ & \\
\hline \multicolumn{5}{|c|}{ Sum of activity energy expenditure in METs-min/week-mean \pm SD } \\
\hline Moderate intensity PA & $1560.2 \pm 1387.9$ & $1684.1 \pm 1618.0$ & $1542.4 \pm 1353.3$ & 0.492 \\
\hline Vigorous intensity PA & $2242.0 \pm 2162.9$ & $2840.1 \pm 2623.7$ & $2156.1 \pm 2078.6$ & 0.033 \\
\hline Sum from all PA & $3802.2 \pm 2743.7$ & $4524.1 \pm 3358.5$ & $3698.5 \pm 2633.0$ & 0.042 \\
\hline
\end{tabular}

${ }^{1}$ Desired shape < perceived shape.

${ }^{2}$ Chi Squares tests for the differences in proportions, T-test for comparison of means (SD). 
TABLE 3 | Partial correlation coefficients (controlling for age) between perceived body image (BI), desired body image (BI), and body dissatisfaction with selected lifestyle behaviors.

\begin{tabular}{|c|c|c|c|c|c|c|}
\hline \multirow[t]{2}{*}{ Variable } & \multicolumn{2}{|c|}{ Perceived BI } & \multicolumn{2}{|c|}{ Desired BI } & \multicolumn{2}{|c|}{ Body Dissatisfaction } \\
\hline & $r$ & $p$-Value & $r$ & $p$-Value & $r$ & $p$-Value \\
\hline Sleep duration (hour/day) & -0.011 & 0.832 & -0.053 & 0.297 & 0.000 & 0.998 \\
\hline Screen time (hour/day) & 0.108 & 0.032 & 0.031 & 0.547 & 0.077 & 0.130 \\
\hline \multicolumn{7}{|c|}{ Physical activity (METs-min/week) } \\
\hline Moderate Physical Activity & 0.051 & 0.311 & 0.028 & 0.580 & 0.042 & 0.404 \\
\hline Vigorous Physical Activity & -0.118 & 0.020 & -0.138 & 0.006 & -0.043 & 0.402 \\
\hline Total Physical Activity & -0.066 & 0.190 & -0.094 & 0.062 & -0.012 & 0.812 \\
\hline \multicolumn{7}{|c|}{ Food frequency intake (day/week) } \\
\hline Breakfast intake & -0.070 & 0.166 & -0.035 & 0.491 & -0.060 & 0.237 \\
\hline Vegetables intake & -0.046 & 0.369 & -0.039 & 0.439 & -0.028 & 0.578 \\
\hline Fruit intake & -0.025 & 0.623 & -0.006 & 0.911 & -0.011 & 0.835 \\
\hline Milk and dairy products intake & 0.010 & 0.840 & -0.031 & 0.544 & 0.036 & 0.480 \\
\hline Sugar-sweetened drinks intake & 0.003 & 0.960 & 0.050 & 0.326 & -0.025 & 0.624 \\
\hline Fast foods intake & -0.044 & 0.385 & -0.019 & 0.708 & -0.052 & 0.301 \\
\hline French fries/potato chips intake & 0.002 & 0.964 & 0.002 & 0.962 & 0.006 & 0.911 \\
\hline Cake/donuts intake & -0.076 & 0.132 & -0.059 & 0.246 & -0.050 & 0.328 \\
\hline Chocolates/candy intake & -0.104 & 0.040 & -0.010 & 0.847 & -0.124 & 0.015 \\
\hline Energy drinks intake & -0.102 & 0.043 & 0.027 & 0.589 & -0.143 & 0.004 \\
\hline
\end{tabular}

intake, eating disorders, and dysfunctional exercise (NeumarkSztainer et al., 2006; Voelker et al., 2015). On the other hand, healthy weight control behaviors were more prevalent among adolescents with lower BD (Lampard et al., 2016).

In our study, PA was not found to predict BD when adjusted for socio-demographic confounders. However this may be due to the homogeneity of the sample in term of PA levels, as the majority of the women were achieving the recommended levels ( $\geq 150 \mathrm{~min} /$ week) of weekly PA (Bull et al., 2020). This is contrary to studies showing PA having a positive and direct effect on BD (Fernández-Bustos et al., 2019) as well as other studies that have consistently shown active subjects to have a more positive BI than sedentary subjects (Bibiloni et al., 2017). This may explain why in our sample, females with longer club memberships showed significantly higher BI satisfaction. It is concerning that a high proportion of normal weight women in this sample (68\%) were also dissatisfied with their body. Dissatisfaction may trigger disordered exercise behaviors and unnecessary attempts at weight control possibly leading to eating disorders. Secondary analysis of this group, however, did not reveal any significant differences in lifestyle habits with their "satisfied" peers.

The current study showed significant associations of BD with reduced dairy products and energy drinks consumption. Findings from a study conducted on Portuguese girls indicated that after adjusting for all demographic and lifestyle factors, only milk intake was negatively associated with BMI and body fat percentage (Abreu et al., 2012). Proper consumption of milk and dairy products can support body mass satisfaction. Other lifestyle behaviors in the present study did not significantly associate with BD. However, a study conducted among Polish adolescents aged 13-19 years demonstrated that being girls, older participants (1719 years old), overweight or obese adolescents and having higher screen time (over $4 \mathrm{~h}$ ) were more likely to be dissatisfied with their body weight (Wawrzyniak et al., 2020).

The present study has several strengths and limitations. Among the strengths of this study is the recruitment of a representative sample of Saudi women from all fitness centers in the city of Riyadh. Second, a reliable and valid lifestyle questionnaire was used that includes PA, sedentary behaviors,

TABLE 4 | Results of multivariable logistic regression for predictors of body image dissatisfaction from lifestyle variables, body size perception, and demographic factors while controlling for age, education level, family income, and father's and mother's education levels.

\begin{tabular}{lccc}
\hline Dissatisfied* & aOR & Cl & $\boldsymbol{p}$-Value \\
\hline Age & 0.98 & $0.29-3.28$ & 0.975 \\
Education level & 0.765 & $0.21-2.73$ & 0.679 \\
Family income & & & \\
$>25,000$ SR** & 1.00 & & \\
15,001-25,000 SR & 1.19 & $0.309-4.576$ & 0.801 \\
15,000 or less & 0.70 & $0.208-2.367$ & 0.569 \\
Father's education & 0.86 & $0.29-2.551$ & 0.786 \\
Mother's education & 1.47 & $0.50-4.330$ & 0.487 \\
BMI & 1.73 & $1.38-2.16$ & $<0.001$ \\
Club membership & & & \\
Less than 1 year** & 1.00 & & 0.041 \\
1-2 years & 0.27 & $0.078-0.948$ & 0.032 \\
Consumption of dairy products/week & 0.79 & $0.643-0.980$ & 0.006 \\
\hline Consumption of energy drinks/week & 0.26 & $0.099-0.679$ & \\
\hline
\end{tabular}

*Reference category is: satisfied.

**Reference group.

aOR, adjusted odds ratio; $\mathrm{Cl}$, confidence intervals. 
sleep, and dietary habits. Third, weight and height of subjects were actually measured and not reported by the participants. On the other hand, some limitations for the present study include the cross-sectional nature of this study which does not allow assumption of causal relationships between the BD, BIP and lifestyle or socio-demographic factors. Second, due to the nature of questionnaires in general, the potential for recall bias cannot be excluded. Third, as the sample came from females who are already participating regularly in fitness centers, it is possible that their BI differs from the general population and thus the study findings may to not be generalized to less active Saudi females. Fourth, BMI was used without accounting for body composition and as this does not distinguish between lean mass and fat mass, we may have misclassified some women who were more muscular. Fifth, the selected BI measures in the present study were based on FRS, which provides a partial assessment of women's body size concerns.

\section{CONCLUSION}

The present study examined the associations of BI perception and $\mathrm{BD}$ with several lifestyle and socio-demographic factors among Saudi women attending fitness centers in Riyadh. The majority of women were overweight or obese, however, nearly half of the sample inaccurately perceived their body shape, mainly underestimating it. The majority of respondents were also dissatisfied with their body shape, even among those in the normal weight category. In addition, among all investigated lifestyle behaviors, only vigorous and total activity energy expenditure in METs-min/week showed significantly lower values for dissatisfied women compared to satisfied participants. However, when adjusting for socio-demographic variables, logistic regression analysis revealed significant associations of BD with higher BMI, shorter membership duration of fitness club, and reduced dairy products and energy drinks consumption. Other lifestyle behaviors did not significantly associate with BD. The results support previous studies which suggest that accuracy of body shape estimation and BD may not be positively associated with healthy lifestyle habits and weight control behaviors. Our results on the associations between $\mathrm{BD}$ and lifestyle behaviors add to those findings from limited studies in the literature. The present findings can inform healthcare providers when

\section{REFERENCES}

Abreu, S., Santos, R., Moreira, C., Santos, P. C., Vale, S., SoaresMiranda, L., et al. (2012). Milk intake is inversely related to body mass index and body fat in girls. Eur. J. Pediatr. 171, 1467-1474. doi: 10.1007/s00431-012-1742-4

Ainsworth, B. E., Haskell, W. L., Herrmann, S. D., Meckes, N., Bassett, D. R. Jr., Tudor-Locke, C., et al. (2011). Compendium of physical activities: a second update of codes and MET values. Med. Sci. Sports Exerc. 43, 1575-1581.doi: 10.1249/MSS.0b013e31821ece12

Albeeybe, J., Alomer, A., Alahmari, T., Asiri, N., Alajaji, R., Alajaji, R., et al. (2018). Body size misperception and overweight or obesity among Saudi college-aged females. J. Obes. 2018:5246915. doi: 10.1155/2018/5246915 intervention strategy is implicated for females with $\mathrm{BD}$, by recognizing the complexity of the associations between $\mathrm{BD}$ and lifestyle behaviors. Future studies should compare associations of $\mathrm{BD}$ with lifestyle behaviors between males and females attending fitness centers and seeking weight loss.

\section{DATA AVAILABILITY STATEMENT}

The raw data supporting the conclusions of this article will be made available by the authors, upon reasonable request.

\section{ETHICS STATEMENT}

The studies involving human participants were reviewed and approved by Princess Nourah Bint Abdulrahman University Ethical Committee-IRB Log Number: 18-0222. The patients/participants provided their written informed consent to participate in this study.

\section{AUTHOR CONTRIBUTIONS}

HA-H, NA, MA, and AA: study concept. NA, HA-H, and LA: statistical analyses. MA, NA, AA, and LA: data collection supervision. NA and HA-H: interpretation of the findings and drafting the manuscript. All authors critically read, revised, and approved the final version of the manuscript.

\section{FUNDING}

This research was funded by the Deanship of Scientific Research at Princess Nourah Bint Abdulrahman University through the Fast-track Research Funding Program.

\section{ACKNOWLEDGMENTS}

We would like to thank all the participants for taking part in this study. Also, special thanks to the following research assistants, who participated in data collection: Sultanah Alsulaiman, Doaa Aljasser, and Shahad Alshalan.

Al-Hazzaa, H., and Al-Ahmidi, M. (2003). A self-reported questionnaire for assessing physical activity in youth 15-25 years: its development, reliability and construct validity. Arab. J. Food Nutr. 4, 279-291. doi: 10.1093/aje/kwh209

Al-Hazzaa, H., and Musaiger, A. (2011). Arab Teens Lifestyle Study (ATLS): objectives, design, methodology and implications. Diabetes Metab. Syndr. Obes. Targets Ther. 4:417.doi: 10.2147/dmso.s26676

Al-Hazzaa, H. M., Abahussain, N. A., Al-Sobayel, H. I., Qahwaji, D. M., and Musaiger, A. O. (2011a). Physical activity, sedentary behaviors and dietary habits among Saudi adolescents relative to age, gender and region. Int. J. Behav. Nutr. Phys. Act. 8:140.doi: 10.1186/1479-5868-8-140

Al-Hazzaa, H. M., Al-Sobayel, H. I., and Musaiger, A. O. (2011b). Convergent validity of the Arab teens lifestyle study (ATLS) physical activity questionnaire. Int. J. Environ. Res. Public Health 8, 3810-3820.doi: 10.3390/ijerph8093810 
Alhussaini, A. A., Alsuwedan, H. I., Alnefaie, H. F., Almubrek, R. A., Aldaweesh, S. A., Anitha, L., et al. (2018). Self-perception of body image among Saudi females at Princess Nourah University, Riyadh, Kingdom of Saudi Arabia. Malays. J. Nutr. 24, 117-123.

Allen, M. S., and Walter, E. E. (2016). Personality and body image: a systematic review. Body Image 19, 79-88.doi: 10.1016/j.bodyim.2016.08.012

Al-Otaibi, H. H., Nassef, S. L., and Raouf, T. A. (2013). Body shape dissatisfaction, weight status and physical activity among a sample university students in Saudi Arabia. Food Nutr. Sci. 04, 616-625.doi: 10.4236/fns.2013.46079

AlTamimi, A. A., Albawardi, N. M., AlMarzooqi, M. A., Aljubairi, M., and Al-Hazzaa, H. M. (2020). Lifestyle behaviors and socio-demographic factors associated with overweight or obesity among Saudi females attending fitness centers. Diabetes Metab. Syndr. Obes. 13, 2613-2622.doi: 10.2147/DMSO. S255628

Anderson, L. A., Eyler, A. A., Galuska, D. A., Brown, D. R., and Brownson, R. C. (2002). Relationship of satisfaction with body size and trying to lose weight in a national survey of overweight and obese women aged 40 and older, United States. Prev. Med. (Baltim) 35, 390-396.doi: 10.1006/pmed.2002.1079

Baranowski, T., Cullen, K. W., Nicklas, T., Thompson, D., and Baranowski, J. (2003). Are current health behavioral change models helpful in guiding prevention of weight gain efforts? Obes. Res. 11(SUPPL. 1), 23S-43S. doi: 10. 1038/oby.2003.222

Ben-Ammar, A. A., and Al-Holy, M. A. (2013). Body image and lifestyle attitudes of female gymnasium users in Saudi Arabia. Nutr. Food Sci. 43, 365-373.doi: 10.1108/NFS-10-2012-0110

Bibiloni, M. D. M., Coll, J. L., Pich, J., Pons, A., and Tur, J. A. (2017). Body image satisfaction and weight concerns among a Mediterranean adult population. BMC Public Health 17:39. doi: 10.1186/s12889-016-3919-7

Bucchianeri, M. M., Arikian, A. J., Hannan, P. J., Eisenberg, M. E., and NeumarkSztainer, D. (2013). Body dissatisfaction from adolescence to young adulthood: findings from a 10-year longitudinal study. Body Image 10, 1-7.doi: 10.1016/j. bodyim.2012.09.001

Bulik, C. M., Wade, T. D., Heath, A. C., Martin, N. G., Stunkard, A. J., and Eaves, L. J. (2001). Relating body mass index to figural stimuli: population-based normative data for caucasians. Int. J. Obes. 25, 1517-1524.doi: 10.1038/sj.ijo. 0801742

Bull, F. C., Al-Ansari, S. S., Biddle, S., Borodulin, K., Buman, M. P., Cardon, G., et al. (2020). World Health Organization 2020 guidelines on physical activity and sedentary behaviour. Br. J. Sports Med. 54, 1451-1462. doi: 10.1136/ bjsports-2020-102955

Clark, H. L., Heileson, J., DeMay, J., and Cole, R. E. (2017). Misperceptions of weight status in military men and women. Mil. Med. 182, e1792-e1798. doi: 10.7205/milmed-d-16-00202

Dorsey, R. R., Eberhardt, M. S., and Ogden, C. L. (2009). Racial/ethnic differences in weight perception. Obesity 17, 790-795.doi: 10.1038/oby. 2008.603

Duong, H. T., and Roberts, R. E. (2014). Perceived weight in youths and risk of overweight or obesity six years later. J. Psychosom. Res. 76, 23-27.doi: 10.1016/ j.jpsychores.2013.11.007

Fan, M., and Jin, Y. (2015). The effects of weight perception on adolescents' weight-loss intentions and behaviors: evidence from the youth risk behavior surveillance survey. Int. J. Environ. Res. Public Health 12, 14640-14668.doi: 10.3390/ijerph121114640

Feng, X., and Wilson, A. (2019). Does dissatisfaction with, or accurate perception of overweight status help people reduce weight? Longitudinal study of Australian adults. BMC Public Health 19:619. doi: 10.1186/s12889-019-6938-3

Fernández-Bustos, J. G., Infantes-Paniagua, Á, Cuevas, R., and Contreras, O. R. (2019). Effect of physical activity on self-concept: theoretical model on the mediation of body image and physical self-concept in adolescents. Front. Psychol. 10:1537. doi: 10.3389/fpsyg.2019.01537

Gouveia, M. J., Frontini, R., Canavarro, M. C., and Moreira, H. (2014). Quality of life and psychological functioning in pediatric obesity: the role of body image dissatisfaction between girls and boys of different ages. Qual. Life Res. 23, 2629-2638.doi: 10.1007/s11136-014-0711-y

Hausenblas, H. A., and Fallon, E. A. (2006). Exercise and body image: a metaanalysis. Psychol. Health 21, 33-47.doi: 10.1080/14768320500105270

Haynes, A., Kersbergen, I., Sutin, A., Daly, M., and Robinson, E. (2018). A systematic review of the relationship between weight status perceptions and weight loss attempts, strategies, behaviours and outcomes. Obes. Rev. 19, 347363.doi: 10.1111/obr.12634

Herman, K. M., Hopman, W. M., and Rosenberg, M. W. (2013). Self-rated health and life satisfaction among Canadian adults: associations of perceived weight status versus. BMI Qual. Life Res. 22, 2693-2705. doi: 10.1007/s11136-0130394-9

Hirshkowitz, M., Whiton, K., Albert, S. M., Alessi, C., Bruni, O., DonCarlos, L., et al. (2015). National sleep foundation's sleep time duration recommendations: methodology and results summary. Sleep Health 1, 40-43.doi: 10.1016/j.sleh. 2014.12.010

Hsu, Y. W., Liou, T. H., Liou, Y. M., Chen, H. J., and Chien, L. Y. (2016). Measurements and profiles of body weight misperceptions among Taiwanese teenagers: a National survey. Asia Pac. J. Clin. Nutr. 25, 108-117. doi: 10.6133/ apjen.2016.25.2.08

Jackson, S. E., Johnson, F., Croker, H., and Wardle, J. (2015). Weight perceptions in a population sample of English adolescents: cause for celebration or concern? Int. J. Obes. 39, 1488-1493.doi: 10.1038/ijo.2015.126

Jankauskiene, R., and Baceviciene, M. (2019). Body image concerns and body weight overestimation do not promote healthy behaviour: evidence from adolescents in Lithuania. Int. J. Environ. Res. Public Health 16, 1-14. doi: 10. 3390/ijerph16050864

Jun, E. M., and Choi, S. B. (2014). Obesity, body image, depression, and weightcontrol behaviour among female university students in Korea. J. Cancer Prev. 19, 240-246.doi: 10.15430/jcp.2014.19.3.240

Kantanista, A., Osiński, W., Borowiec, J., Tomczak, M., and Król-Zielińska, M. (2015). Body image, BMI, and physical activity in girls and boys aged 14-16 years. Body Image 15, 40-43.doi: 10.1016/j.bodyim.2015.05.001

Lampard, A. M., Maclehose, R. F., Eisenberg, M. E., Larson, N. I., Davison, K. K., and Neumark-Sztainer, D. (2016). Adolescents who engage exclusively in healthy weight control behaviors: who are they? Int. J. Behav. Nutr. Phys. Act. 13, 1-10. doi: 10.1186/s12966-016-0328-3

Lawler, M., and Nixon, E. (2011). Body dissatisfaction among adolescent boys and girls: the effects of body mass, peer appearance culture and internalization of appearance ideals. J. Youth Adolesc. 40, 59-71.doi: 10.1007/s10964-009-9500-2

López, M. I., and Rodríguez Cabeo, D. (2020). Body image of Spanish children and adolescents. Differences by diet and physical activity. Atena J. Sports Sci. 2:5.

Lynch, E., Liu, K., Wei, G. S., Spring, B., Kiefe, C., and Greenland, P. (2009). The relation between body size perception and change in body mass index over 13 years. Am. J. Epidemiol. 169, 857-866.doi: 10.1093/aje/kwn412

Matthiessen, J., Biltoft-Jensen, A., Fagt, S., Knudsen, V. K., Tetens, I., and Groth, M. V. (2014). Misperception of body weight among overweight Danish adults: trends from 1995 to 2008. Public Health Nutr. 17, 1439-1446.doi: 10.1017/ S1368980013001444

McElhone, S., Kearney, J. M., Giachetti, I., Zunft, H. J. F., and Martínez, J. A. (1999). Body image perception in relation to recent weight changes and strategies for weight loss in a nationally representative sample in the European Union. Public Health Nutr. 2, 143-151.doi: 10.1017/S1368980099000191

Memish, Z. A., El, Bcheraoui C, Tuffaha, M., Robinson, M., Daoud, F., Jaber, S., et al. (2014). Obesity and associated factors-Kingdom of Saudi Arabia, 2013. Prev. Chronic Dis. 11:E174. doi: 10.5888/pcd11.140236

Miller, E. C., Schulz, M. R., Bibeau, D. L., Galka, A. M., Spann, L. I., Martin, L. B., et al. (2008). Factors associated with misperception of weight in the stroke belt. J. Gen. Intern. Med. 23, 323-328.doi: 10.1007/s11606-007-0499-3

Monteagudo, Sánchez C, Dijkstra, S. C., and Visser, M. (2015). Self-perception of body weight status in older Dutch adults. J. Nutr. Health Aging 19, 612-618.doi: 10.1007/s12603-015-0486-z

Musaiger, A., bin Zaal, A., and D’Souza, R. (2012). Body weight perception among adolescents in Dubai, United Arab Emirates. Nutr. Hosp. 27, 1966-1972.

Musaiger, A. O. (2014). Body size preferences among young women in five Arab countries: a cross-cultural study. Int. J. Adolesc. Med. Health 26, 417-421.doi: 10.1515/ijamh-2013-0317

Neumark-Sztainer, D., Paxton, S. J., Hannan, P. J., Haines, J., and Story, M. (2006). Does body satisfaction matter? five-year longitudinal associations between body satisfaction and health behaviors in adolescent females and males. J. Adolesc. Health 39, 244-251.doi: 10.1016/j.jadohealth.2005.12.001

Neves, C. M., Cipriani, F. M., Meireles, J. F. F., Da Rocha Morgado, F. F., and Ferreira, M. E. C. (2017). Body image in childhood: an integrative literature 
review. Rev. Paul. Pediatr. 35, 331-339. doi: 10.1590/1984.0462/;2017;35;3; 00002

Paans, N. P. G., Bot, M., Brouwer, I. A., Visser, M., and Penninx, B. W. J. H. (2018). Contributions of depression and body mass index to body image. J. Psychiatr. Res. 103, 18-25.doi: 10.1016/j.jpsychires.2018.05.003

Rancourt, D., Thurston, I., Sonneville, K., Milliren, C., and Richmond, T. (2017). Longitudinal impact of weight misperception and intent to change weight on body mass index of adolescents and young adults with overweight or obesity. Eat. Behav. 27, 7-13. doi: 10.1016/j.eatbeh.2017.08.002

Rejeski, W. J., and Fanning, J. (2019). Models and theories of health behavior and clinical interventions in aging: a contemporary, integrative approach. Clin. Interv. Aging 14, 1007-1019.doi: 10.2147/CIA.S206974

Robinson, E., Hunger, J. M., and Daly, M. (2015). Perceived weight status and risk of weight gain across life in US and UK adults. Int. J. Obes. 39, 1721-1726.doi: 10.1038/ijo.2015.143z

Sabiston, C., Pila, E., Vani, M., and Thogersen-Ntoumani, C. (2019). Body image, physical activity, and sport: a scoping review. Psychol. Sport Exerc. 42, 48-57. doi: 10.1016/j.psychsport.2018.12.010

Sampasa-Kanyinga, H., Hamilton, H. A., Willmore, J., and Chaput, J. P. (2017). Perceptions and attitudes about body weight and adherence to the physical activity recommendation among adolescents: the moderating role of body mass index. Public Health 146, 75-83.doi: 10.1016/j.puhe.2017.01.002

Sonneville, K. R., Thurston, I. B., Milliren, C. E., Gooding, C., and Richmond, T. K. (2016). Weight misperception among young adults with overweight/obesity associated with disordered eating behaviors. Int. J. Eat. Disord. 49, 937-946.doi: 10.1002/eat. 22565

Stunkard, A. J., Sorenson, T., and Schulsinger, F. (1983). "Use of the Danish adoption register for the study of obesity and thinness," in The Genetics of Neurological and Psychiatric Disorders, eds S. Kety, L. R. Rowland, L. Sidman Richard, and W. Matthysse Steven (New York, NY: Raven press), $115-120$.

Voelker, D., Reel, J., and Greenleaf, C. (2015). Weight status and body image perceptions in adolescents: current perspectives. Adolesc. Health Med. Ther. 6, 149-158.doi: 10.2147/AHMT.S68344

Wawrzyniak, A., Myszkowska-Ryciak, J., Harton, A., Lange, E., Laskowski, W. Hamulka, J., et al. (2020). Dissatisfaction with body weight among polish adolescents is related to unhealthy dietary behaviors. Nutrients 12:2658.doi: $10.3390 /$ nu 12092658

World Health Organization (2000). Obesity: Preventing and Managing the Global Epidemic. Report of WHO Consultation on Obesity. Geneva: World Health Organization.

Yager, Z., and O'Dea, J. A. (2008). Prevention programs for body image and eating disorders on University campuses: a review of large, controlled interventions. Health Promot. Int. 23, 173-189. doi: 10.1093/heapro/dan004

Conflict of Interest: The authors declare that the research was conducted in the absence of any commercial or financial relationships that could be construed as a potential conflict of interest.

Copyright (C) 2021 Albawardi, AlTamimi, AlMarzooqi, Alrasheed and Al-Hazzaa. This is an open-access article distributed under the terms of the Creative Commons Attribution License (CC BY). The use, distribution or reproduction in other forums is permitted, provided the original author(s) and the copyright owner(s) are credited and that the original publication in this journal is cited, in accordance with accepted academic practice. No use, distribution or reproduction is permitted which does not comply with these terms. 